\title{
Real Time Location based Tracking using WIFI Signals
}

\author{
Atul Gosai. Ph.D \\ Assistant Professor, \\ Department of Computer Science, Saurashtra \\ University, Rajkot
}

\author{
Rushi Raval \\ Assistant Professor, \\ Department of M. Sc. (IT), GK\&CK Bosamia \\ College, Jetpur (Affiliated with Saurashtra \\ University)
}

\begin{abstract}
Now-a-days the difficult to tracking the mobile devices has become an issue. Various needs are arising for finding out ways of tracking mobile devices. In this research paper included different algorithm for location tracking. The activity of tracking includes learning and inference, sensing. Different algorithms have different mechanisms based on which the tracking is made possible. For tracking a device in nearby place efforts required are less than from far-away places. Algorithms include their own mechanisms for tracking the devices easily. Some of the algorithms are simple in nature while others are complex. The cost incurred for tracking devices differs when used via wireless against wired networks. Wireless technology is beneficial for tracking the devices in close areas easily. Wire-less technology such as WI-FI becomes very helpful in such cases. For tracking devices in indoor places the system named WITS (Wireless Indoor Tracking System) is used. WLAN based location tracking algorithms is categorized into two types: deterministic and probabilistic. In this paper algorithm such as Bayesian algorithm, nearest neighbor algorithm, Historybased tracking algorithm, H.M.M. (Hidden Markov model), RADAR etc. is explained. The use of wireless 802.11 frameworks is done to locate the devices. Moreover the WI-FI technology can also be used in forest area for tracking animals. WI-FI signals are much useful in places where the wired connections are not possible to set up. Despite of the use of WI-FI signals in location tracking, they are also used in tracking Bar Code stickers. Some of the WI-FI based techniques are only software based so it decreases the cost of hardware maintenance. Radio-frequency based tracking in WLAN signals has gained more and more popularity in recent years. In early days the WLAN was used to track only static devices but later on by making advances in the technology it was possible to track the moving devices as well.
\end{abstract}

\section{Keywords}

WI-FI Location tracking, Bayesian algorithm, Wireless LAN, Wireless Indoor Tracking System (W I T S), Hidden Markov Model (H M M)

\section{INTRODUCTION}

Wi-Fi based systems can potentially work both outdoors and indoors. Example, RADAR is an indoor location system based on Wi-Fi signals [1] [5]. It uses two methods to calculate locations from signal information. The first is to use a signal propagation model, which quantifies how wireless signal fluctuates with distance. Then it estimates the distances from multiple access points from signal strengths.

Triangulation can then be used to determine the location. However, signal strength is influenced by a number of factors other than distance, including obstacles, replication, and refraction, so in practice it is virtually impossible to obtain an accurate propagation model.
As a consequence, this method can only provide coarse location information. The second method is based on fingerprinting. This method works by building a database that maps each location cell to the signal statistics measured at that cell. After the database is built, location can be determined by mapping the signal information back

Fingerprinting can give good location accuracy, however, requires intense manual work to build the mapping The critical part of the place lab system is to build beacon databases that record the locations of the Wi-Fi access points. These location data may come from the Wi-Fi deployment specifications, or from the war-drivers who drive around recording both Wi-Fi signals and GPS measurements.

The place lab system can cover indoor places [12] and many urban canyons where GPS does not work. It does not require any specific location hardware and thus have a cost advantage. However, the location information is less accurate and less reliable, and maintaining the up-to-date beacon databases is a very challenging task.

Wi-Fi localization is used for determining the physical location of a mobile device from the wireless signals, has been made known to be a precise method of indoor and outdoor localization and a great building block for location aware application [4]

The use of wireless signal strength information to localize mobile devices has gained significant interest in several research communities. This is mainly due to the increasing availability of $802.11 \mathrm{Wi}-\mathrm{Fi}$ networks and the importance of location information for applications such as activity recognition.

In recent years, the problem of estimating a person's location has gained interest in several research communities. The centrality of location information to such tasks as activity recognition, surveillance, and context-aware computing can be seen in many applications.

Therefore, it can hardly be applied at a large scale. One large scale location system based on Wi-Fi signals is the place lab, which works both indoors and outdoors. The place lab emphasizes the coverage of the system rather than the accuracy.

The wide spread of mobiles as handheld devices would naturally lead to various innovative applications that makes use of their ever increasing presence in our day-to-day life. One such application is location-tracking in indoor environments [2]. In this paper we discuss the implementation of a simple and cost effective system that assists users in tracking colleagues and friends within a campus environment. 


\section{EVOLUTION OF WIRELESS TRACKING SYSTEM}

Previous years have proved to be the rising popularity of sensor and sensor network technologies, sustaining central practical applications. One of the essential issues is how to exactly locate a user with few labeled data in a wireless sensor network [11]; the major trouble arises from the need to label large quantities of user location data, which in turn requires information about the locations of signal transmitters or entrance points.

Mobile technology is continuously and rapidly developing over the earlier years. Not only the cellular grid that has been improved but the cellular phone unit or mobile phone itself has also been greatly improved with state-of-the-art technology and enhanced features.

Mobile phones are developed to be faster with better and higher CPU speed and larger memory storage. The devices are designed to be more modern and more fashionable looking with additional features like touch-screen, larger screen, and built-in high resolution/frame camera. They are also equipped with various means of connection such as Bluetooth and WiFi.

The mobile phone operating systems are also more capable of handling quicker, larger and more tasks with variety of choices from many famous software developers to choose. Example: Symbian by Nokia, Windows Mobile by Microsoft, i-Phone by Apple and Android by Google.

Wi-Fi is considered a standard feature for most mobile phones now-a-days. Mostly authors argue that since Wi-Fi (Wireless Fidelity) is widely deployed in buildings such as universities, colleges, hotels, hospitals, airports and metro/rail stations, it makes sense to make use of this technology in support of costeffective location identification or positioning solutions.

One potential area of mobile commerce development is in location-based services (LBS). By the end of September 2002, there were an estimated 605.6 million Internet users worldwide In June 2014, the global number of mobile phone users reached one billion for the first time. Together, the convergence of these technologies promises to bring further services. Worldwide, revenues from mobile commerce i.e. transactions over wireless telecommunications network are expected to exceed $\$ 75$ billion by 2014 .

Location techniques operate in two steps: signal measurements and location estimate computation based on the measurements which may be carried out by the user equipment or the telecommunications network. Wi-Fi signals are information carriers among a transmitter and the receiver. Wi-Fi can also encompass our senses, enabling us to see movable objects through walls and behind closed doors. Therefore, we can use such signals to recognize the number of people in a closed room and their relative locations. It's also feasible to identify simple gestures made behind a wall, and combine a series of gestures to converse messages to a wireless receiver devoid of carrying any transmitting device.

Wi-Fi network is basically depends on Wi-Fi signal types and Wi-Fi signal reporting. Opened or closed area, building with walls and equipment, external antenna, etc., all of these can affect the quality of Wi-Fi signal since it is a low frequency radio signal that cannot penetrate metal, water and other materials easily. Normally, Wi-Fi network range is about 75$150 \mathrm{ft}$. Many mobile devices today are well equipped with $\mathrm{Wi}$-Fi to enable them to connect to the internet easily.
The latest method of tracking and locating of client-based mobile phone which will eliminate the limitation of the inbuilt GPS phones, the term Wi-Fi was created by an organization called the $\mathrm{Wi}-\mathrm{Fi}$ association to declare products that belong to a class of wireless local area network (WLAN) device based on the IEEE 802.11 standard. A product that passes the association test is given the tag. Any Wi-Fi licensed devices are able to connect and communicate with one another by mean of Wi-Fi.

It has been known that GPS usage is limited in buildings and places unreachable by satellite signal. In order to find better and more precise results, the method of assimilating Wi-Fi signal, cell positions and vector calculation to track and locate mobile instruments are introduced

Moreover, it's also beneficial to develop this method to be applied with other utility programs such as: delivery truck tracking system or personal locating system via mobile phone.

\section{WIRELESS INDOOR TRACKING SYSTEM}

All Wireless Indoor Tracking System (WITS) [15] is a pure software system, which is used to locate and track mobile devices inside a building. It implements the use of existing WLAN infrastructure and uses wireless adapter as the sensor. The system is implemented using $\mathrm{C}++$, and also composed of server, client and builder.

WITS server is a kind of dedicated computer which is running the location determination algorithm. It receives tracking request from WITS client, estimates its real time location using tracking algorithm, and returns the location information back to WITS client. The WITS client is installed on the mobile device. The current received signal strength, sends tracking request with the received signal strength to W I T S server, and shows the estimated location on the map after receiving reply from WITS server is measured.

WITS client is also able to register in the server that it wants to be tracked so that other person is able to know its position. Before tracking the mobile clients, the RSS radio map must be built using a calibration process, which is the job of WITS builder.

WITS builder will collect samples of signal strength at various locations in the building and sends them to WITS server, which will store them in the RSS database and build the RSS radio map. RSS database is implemented using Microsoft Access. The basic of the tracking system is to be retrieve signal strength values of the visible access points accurately. Source code of Wireless Research API from University of California, which implements the function, is integrated into WITS. It works on Windows XP platform and is independent in areas such as wireless adapters.

The system works in client-server architecture, and uses history based algorithm to estimate the locations of the mobile clients. Design of the building and Bluetooth are incorporated to improve the accuracy. 


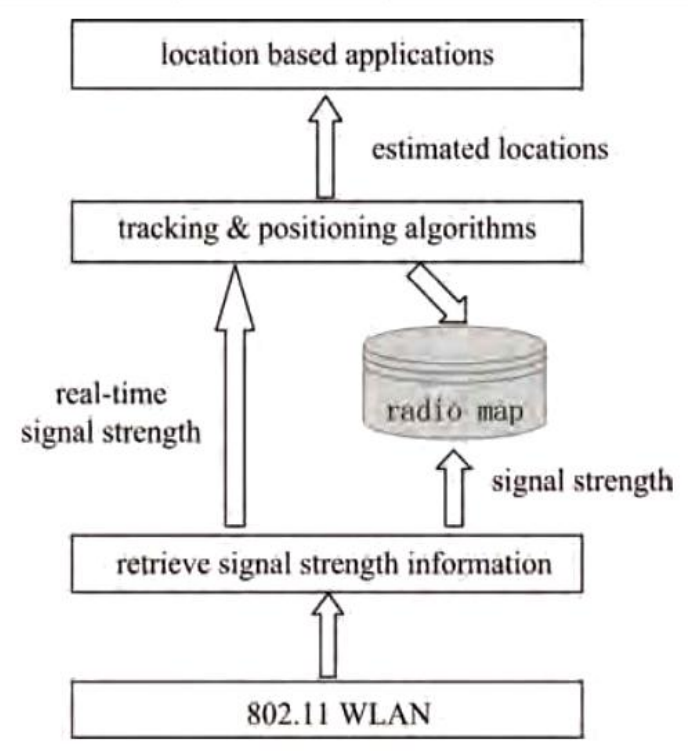

Fig.1: Working steps of WITS

System architecture of WITS

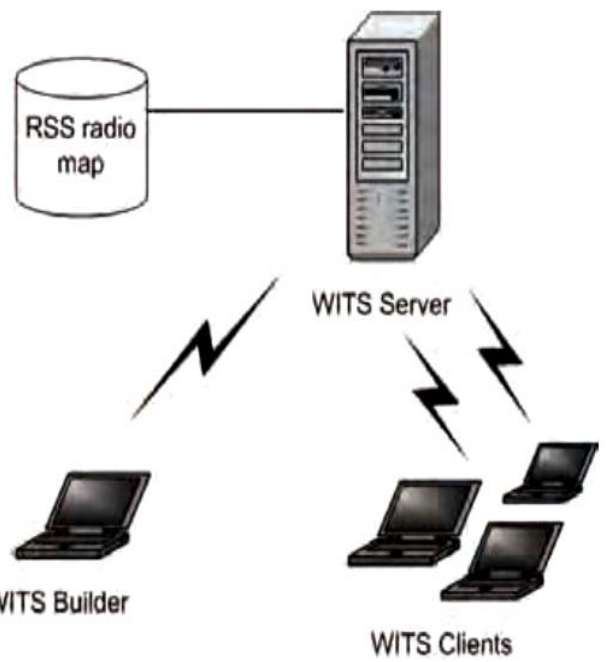

Fig.2: Architecture of WITS

\section{Category of WITS algorithms}

According to the principle of choosing the most suitable locations of the mobile devices, WLAN-based location determination algorithms are categorized into two groups: deterministic and probabilistic algorithms. The eldest and the most basic deterministic location determination algorithm is the nearest neighbor algorithm, which is used in RADAR [5] [13] system Probabilistic location determination and it makes use of Bayesian law [6].

\section{1) Nearest Neighbour Algorithm}

The nearest neighbor algorithm [20] provides the basic idea of deterministic WLAN-based positioning. During the process of calibration, the system collects multiple samples of signal strength footprints at the calibrated locations and also stores the signal strength's mean value of all the calibrated location in the RSS radio map. In the tracking phase, the current received signal strength is compared with the mean values in the RSS radio map. The record that matches the current received signal strength best is chosen.

The idea is to calculate the Euclidean distance between the currently received signal strength and the records in the RSS radio map and choose the one which will be minimizing Euclidean distance.

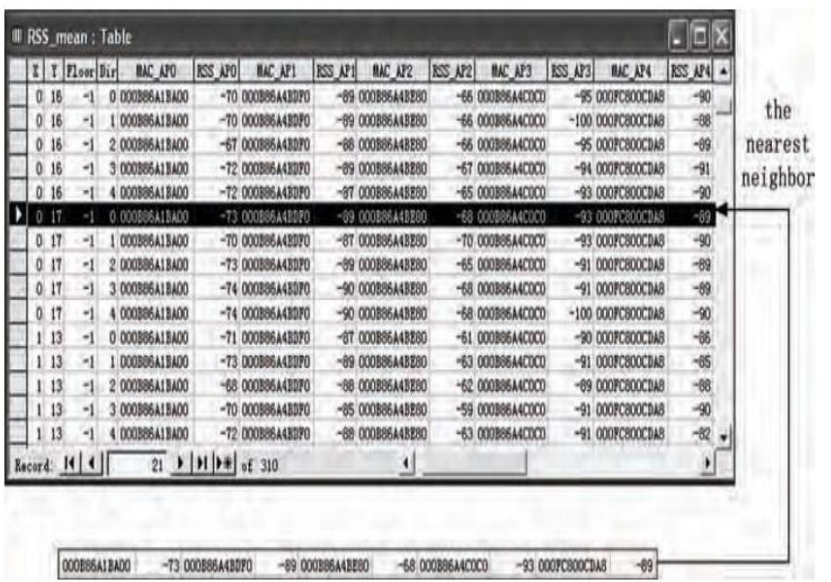

Fig.3: Table of Nearest Neighbor Algorithm

As shown in the figure above, the nearest neighbor algorithm uses a Map for keeping track of the status of the users in a particular location. There is a calibration process in which the tracks of all users are kept so that the location is achieved easily.

2) Bayesian Algorithm

Bayesian algorithm [6] is the basic algorithm used for most probabilistic location determination algorithm. It has the same calibration phase as the nearest neighbor algorithm, but it also makes use of Bayesian law to choose the most likely location of the mobile device.

3) History-based tracking algorithm

As observed from above study, the nearest neighbour algorithm and Bayesian algorithm make use of only the current received signal strength for estimating the unknown location. Also the user cannot jump large distances, WITS uses history based algorithm [17], i.e. a series of consecutive signal strength, for location determination.

Depending on whether the nearest neighbour algorithm or Bayesian algorithm is used to choose the most likely neighbours, the history-based algorithm may be either deterministic or probabilistic.

The following steps must be followed to track any person who is moving:

1. When the user is walking, real-time signal strength is to be collected on periodic basis.

2. Use the nearest neighbour algorithm (deterministic) or Bayesian algorithm (probabilistic) to find the $\mathrm{k}$ most suitable locations in the RSS radio map, known as the $k$ nearest neighbour. Here $\mathrm{K}$ is a predefined parameter.

3. In history vector of $k$ nearest neighbour, the column of the oldest $k$ nearest neighbour is deleted and the column of the 
newest $k$ nearest neighbour is to be added, so that the depth of the history vector keeps $h$.

4. Then, Calculate the shortest path from the last estimated location to the newest $k$ nearest neighbour in to the history vector. The distance between the two adjacent points is called the physical distance.

5. The end point of the shortest path is known as the current estimated location of the mobile device.

6. The following steps are to be repeated, until the client stops tracking process.

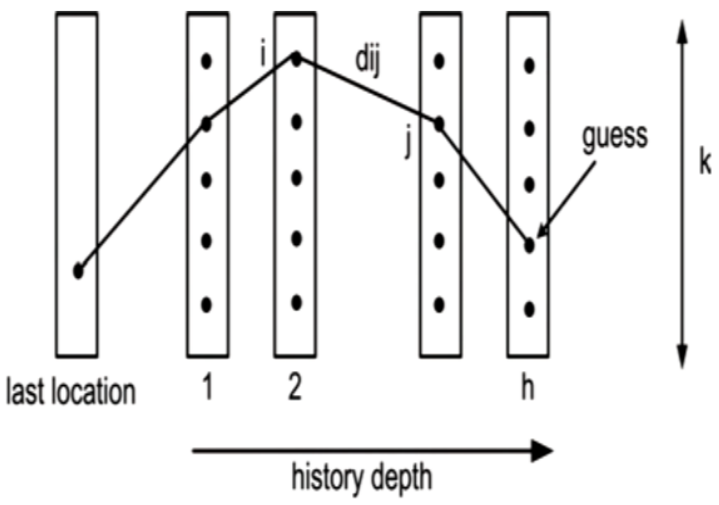

Fig.4: History based Tracking system [17]

As shown above in the figure, the history-based algorithm as the name specifies is used to keep track of the locations of the users who are moving in far-away distances and need to be tracked. As compared to the other two algorithms, this algorithm is more beneficial for users who travel long distance.

\section{Bluetooth support}

Bluetooth is also used in this system as the asset for WLAN tracking technology. Various Bluetooth access points are kept in fixed places and their location information is stored in the Bluetooth table inside the database.

If the mobile device is Bluetooth enabled, it will scan for discovering the visible Bluetooth access points. Based on the Bluetooth access points discovered, the system determines the approximate area that the mobile device is using. The final Gained location of the mobile device is the combination of the estimations from 802.11 WLAN and Bluetooth.

\section{LOCADIO SYSTEM}

For inferring the motion and location from Wi-Fi signal strengths the system which is used, is called LOCADIO (LOCation from $r A D I O$ ) [19]. It uses a simple variance measure as input to a two-state hidden Markov model (HMM) [18] for classifying a client as either still or moving. The final observation that was obtained was when a Wi-Fi receiver is moving, the signal strengths it receives are noisier than when it is not moving.

The main goal for inferring location was to exploit prior assumptions about a person's motion to increase the accuracy and minimize calibration effort. Motion is one of the most important parts of a user's context.
It was studied that it might be possible to classify a user as either still or moving based on Wi-Fi signal strength features. Such a conjecture was supported by the qualitative observation that signal strengths from APs appear to jump around more dangerously when the device is in motion and when the device is still [14].

The basic approach of the system is to classify a user either still or moving is based on the variance of a temporarily short history of signal strengths from the currently strongest access points. It was also found that this classification transitioned too often between the two states, so we smoothed the classifications over time with a two-state HMM

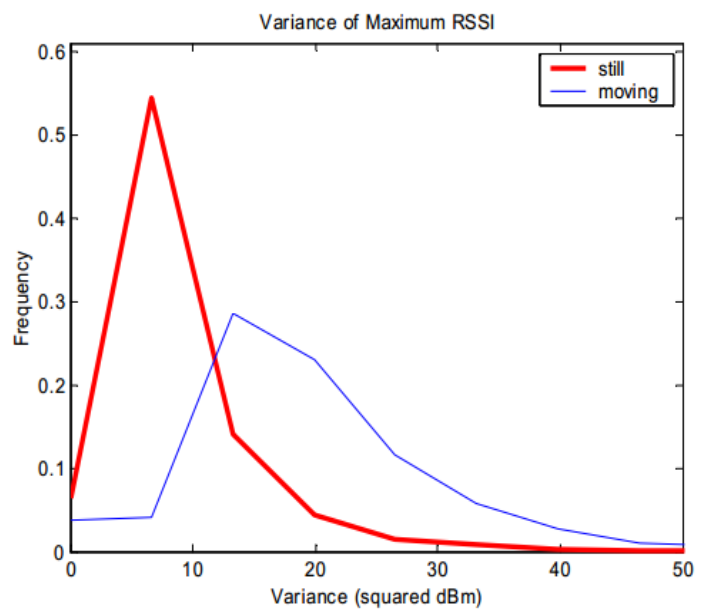

Fig.5: HMM Model [18]

As shown in the figure above, the HMM model makes clear about idea about the location of the user in any area. The red lines are shown for still positions and the blue line is shown for the moving users.

From the above figure the idea about the accuracy of tracking positions for users is easier if the user does not change his/her position and stays still. Whereas when the users keep on changing their locations the accuracy is not maintained much.

It's also not possible to track the users who are moving constantly in different places. So this model proves to be beneficial only if the user is still in one position.

LOCADIO [19] computes whether or not a device is in motion by observing the fluctuations of $\mathrm{Wi}-\mathrm{Fi}$ signal strengths, solving the inferences with an HMM and simple prior assumptions on how the peoples move.

The location part of LOCADIO is derived from a principle model that accounts for the building's layout, expected pedestrian speeds, and probabilistic signal strength signatures. While applied this framework to 802.11 signals, it could be easily applied to other types of location sensing as well as serving as the platform for sensor fusion [11] [12].

\section{Cell Location Positioning Techniques}

This technique works by identifying the cell of the network in which the handset is operating (the "cell of origin"). Cell of origin (COO), sometimes called Cell-id, is the main technology widely deployed in wireless networks today. It requires no modification to handsets or networks since it uses the mobile network base station as the location of the caller. However, although locating the caller is fast typically around three seconds accuracy is limited. 
Positioning accurateness depends on the dimension of the cell and techniques used for enhancing location calculation, such as user self-locating (use landmarks and addresses to improve their positioning precision) and propagation time measurements [14].

Navigation and tracking form a core segment of the emerging LBS market. In the United States, the average person spends 500 hours per year in an automobile. Though, only 100,000 of the 146 \$ registered cars in the United States and $20 \%$ of fleet trucks are equipped for telematics, i.e., LBS for automobiles. With the wide-spread deployment of services, the telematics/mobile market could expand considerably in the next few years [10].

Location technologies can play an important part in logistics. Taxis are being equipped with automatic vehicle location devices, allowing the fleet dispatch system to automatically select the taxi closest to the pickup location [7]

Israel, mobile operator offers a wireless workforce function, using CT Motion's Celebrity platform, which includes location technology based on EOTD. Orange can offer companies the ability to monitor the movements of their workforce throughout the country to an accuracy of up to 100 meters.

In December 2000, 8 leading companies involved in the Wireless location industry in the US, Canada, and Europe CellLoc, SignalSoft, GoAmerica Communications, Cambridge Positioning Systems, Zero Knowledge Systems, Index only Technologies, iProx, and ViaVis Mobile Solutions established another industry group, the Wireless Location Industry Association (WLIA).

\section{REAL TIME LOCATION SYSTEM}

Real time location systems (RTLS) [13] are build using the presence of Access Points (APs) or Location Receivers that are able to detect the tags and Wireless Fidelity (Wi-Fi) devices in the near-by areas.

Two different methods can be used for locating an object:

\section{- Time Difference on Arrival method (TDoA)}

\section{- Received Signal Strength Indicator method (RSSI)}

In the idea of TDoA [3], it calculates the position of an object by measuring the time taken from a packet being sent from a transmitter device to being received by a receiver. By means of Wi-Fi, a client transfers out a time embossed signal which is received by the APs. From the dissimilarity in time between sending and receiving the signals, the distance between the $\mathrm{AP}$ and the client can be designed. When the 3 access points are been used, by the principle of triangulation, it's possible to establish location of a client to an accuracy of less than 5 meters.

RSSI, the client device measures the signal strength of the access points, sends the values to a server. If least 3 values are sent the server can constantly calculate the location of the client.

RSSI performs healthier in indoor walled environments, e.g. Hospitals, while TDoA has advantages in unobstructed, outdoor environments, Example: shipping yards. With TDoA the access points need to be particularly designed to be able to compute the time variations.

\section{LANDMARC System}

The LANDMARC system [16] uses dynamic signals as a reference tags to dynamically construct and update radio map. It relieves the effects caused by the variation in Radio Frequency (RF) [8] [9] [13] signal strength. The method first calculates the distances among the signal-strength vectors received from the tracking tags and those from dissimilar reference tags, respectively. It then uses $\mathrm{k}$ nearest reference tags' co-ordinates to compute the approximate co-ordinate of the tracking tag.

\section{CONCLUSIONS}

In this paper, Different techniques stated for location based tracking and also concentrating on a mass usage of wireless WI-FI signals for location tracing. WI-FI signals allowing a immense field of services to track the user's location, At the time of users are going to be tracked, in that scenario the distance is a major issue to locate the location. Track the users' who are very close enough is an easiness task as comparing to the users' who are located at a far distance going to be tracked.

Numerous algorithms are described in this research paper to track the users' location with use of wireless WI-FI signals. The algorithms are such as: Bayesian Algorithm, Historybased Algorithm, Nearest Neighbor Algorithm, HMM model etc. These all specified algorithms have their own criteria to tracking the user and are helpful for tracking the locations in an easy manner. In day-to-day to life, we are able to facilitate this various kinds of algorithms for location tracking of users' for their safety or finding a place with usage of wireless WIFI signals.

\section{REFERENCES}

[1] F. Barcelo1-Arroyo et al, Indoor Location for Safety Applications using Wireless Networks, in Proceeding of the 1st ERCIM Workshop on Mobility, Portugal, 2014.

[2] A. Haeberlen, E. Flannery, A.M. Ladd, A. Rudys, D.S. Wallach, and L.E. Kavraki, Practical robust localization.

[3] Gardner, W.A., Chih-kang Chen, Signal-selective timedifference-of-arrival estimation for passive location of man-made signal sources in highly corruptive environments. I. Theory and method, Signal Processing, IEEE Transactions on Vol: 40, Issue: 5, August, 2002

[4] Tseng, Y. C., Wu, S. L., Liao, W. H., and Chao, C. M. IEEE Computer (34:6), February 2014, pp. 46-52.

[5] Tracking system in Proceedings of IEEE Infocom.

[6] Fox, D.; Hightower, J.; Liao, L.; Schulz, D.; and Borriello, G. 2014, Bayesian filtering for location estimation, IEEE Pervasive Computing Magazine.

[7] Haeberlen, A.; Flannery, E Ladd, A.; Rudys, A.; Wallach, 10th ACM International Conference on Mobile Computing and Networking.

[8] Krishnan, P., Krishnakumar, A.; Ju, W.H.; Mallows, C.; and Gani, S. 2014. Location estimation assisted by stationary emitters for indoor RF wireless networks. In Proc. of the IEEE Infocom

[9] Ladd, A.; Bekris, K.; Marceau, G.; Rudys, A.; Kavraki, L.; and Wallach, D. 2002. Robotics-based location sensing using Wireless Ethernet, In Proceedings of MOBICOM2002

[10] LaMarca, A.; Chawathe, Y.; Consolvo, S.; Hightower, J. Smith, I.; Scott, J.; Sohn, T.; Howard, J.; Hughes, J.; Potter, F.; In International Conference on Pervasive 
Computing, Munich, Germany, 8-13 May 2005, pp. 116133.

[11] Lauritzen, S. 1996. Graphical Models Oxford University Press, Liao, L.; Fox, D: Hightower, J.; Kautz, H.; and Schulz, D. 2014. Voronoi tracking: Location estimation using sparse and noisy sensor data. In Proc. of the IEEE/RSJ International Conference on Intelligent Robots and Systems (IROS)

[12] E. Foxlin2, "Pedestrian Tracking with Shoe-Mounted Inertial Sensors.

[13] P. Bahl, V. Padmanabhan, RADAR: an in-building RFbased user location and tracking system, in Proceedings of the IEEE Infocom 2000.

[14] A. Carlotto, M. Parodi, C. Bonamico, F. Lavagetto, M. Valla Proximity classification for mobile devices using Wi-Fi environment similarity.

[15] R. Zhou, Wireless Indoor Tracking System (WITS), Communication systems/Computing Centre, University of Freiburg
[16] L. Ni, Y. Liu, Y. Lau, and A. Patil, "LANDMARC: Indoor Location Sensing using Active RFID," Proceedings First IEEE International Conf. Pervasive Computing and Communication, pp. 407-416, Mar. 2003.

[17] Eoghan Furey, Kevin Curran, Paul Mc Kevitt , HABITS: A History Aware Based Wi-Fi Indoor Tracking System, PGNET conference, 2008.

[18] G.A. Fink., Markov Models for pattern recognition, Advances in Computer vision and pattern recognition, DOI 10.1007/978-1-4471-6308-4_5, Springer-Verlag, London, 2014.

[19] John Krumm, Eric Horvitz, Microsoft research, LOCADIO: Inferring Motion and Location from Wi-Fi Signal Strengths, August 22-26, 2004, Boston, MA, USA

[20] Scott cost, Steven salzberg, A weighted nearest neighbor algorithm for learning with symbolic future, Machine Learning, 10, 57-78. 